order law, as is not surprising in a reactant of this complexity. Thus, during the first one-third of the reaction in I-chloro-2:4-dinitrobenzene, the rate of evolution of gas shows only a slight fall, corresponding to a gradual increase of about 25 per cent in the rate constant; whereas during the last quarter, the rate constant falls to about 60 per cent of its value at the intermediate stage. If one specifies the rate of the reaction, perhaps rather arbitrarily, by the velocity constant prevailing over the middle of its course, one finds that the rates at $175^{\circ} \mathrm{C}$. in the three solvents show a spread of only 20 per cent, a sufficiently close agreement to suggest that the solvents are inert towards nitrocellulose.

In 1-chloro-2:4-dinitrobenzene, the rates at six temperatures between $165^{\circ} \mathrm{C}$. and $200^{\circ} \mathrm{C}$. lead to the following equation for the velocity constant :

$$
k\left(\text { sec. }^{-1}\right)=10^{18 \cdot 0} \exp \left(-43 \cdot 0 \times 10^{3} / R T\right) .
$$

The average deviation, without regard to sign, between the observed rates and those calculated from this equation is 7 per cent. It is unlikely that this preliminary value of $43.0 \mathrm{kcal}$. for the activation energy is in error by more than 2 kcal. It is therefore doubtful whether nitrocellulose is behaving as a normal nitrate when judged by Phillips's criterion of the activation energy. The reason for the high value of $10^{18.0}$ for the $B$ factor is not certain, but even higher values have been recorded before, and Wilfong, Penner and Daniells probably seriously under-estimate the rate of decomposition in this temperature-region when they assume in effect that $B$ has its 'normal' value of $10^{13}$.

In 1-chloro-2:4-dinitrobenzene and benzophenone solutions, about five molecules of gas come from each sub-molecule of nitrocellulose. This yield of gas seems at first sight small; but it has been observed before ${ }^{5}$ that much, perhaps one-half, of the products of pyrolysis of nitrocellulose is involatile. From l-nitronaphthalene solutions, however, the yield is only $3 \cdot 5$ molecules per sub-molecule. Here the solvent presumably absorbs some of the products, but this need not invalidate the rates measured for its solutions. The reaction products have not been analysed.

Research Department, R. D. SMITH Nobel Division,

Imperial Chemical Industries, Ltd.,

Stevenston, Ayrshire.

$$
\text { May } 14 .
$$

${ }^{1}$ Wilfong, R. E., Penner, S. S., and Daniells, K., J. Phys. Coll. Chem. 54, 863 (1950).

8 Phillips, L., Nature, 160, 753 (1947).

s Robertson, A. J. B., J. Soc. Chem. Indust., 67, 221 (1948).

4 Robertson, A. J. B., Trans. Farad. Soc., 45, 85 (1949).

${ }^{5}$ Desmaroux, J., Mem. des Poudres, 29, 189 (1939).

\section{Paper Chromatographic Separation of Barbituric Acids}

The barbituric acid derivatives have a characteristic absorption spectrum in the ultra-violet, and this is the basis of the most reliable methods for their quantitative determination. Small changes of the wave-length at which maximal absorption is found and changes in the absorption curve with varying $p H$ usually are considered to be the means by which the different members of the group can be identified. But these methods have been found troublesome and unreliable. However, as the strength of absorption differs quite appreciably from one member to another, the identification of the derivative is necessary for a quantitative determination.

It seemed theoretically that paper chromatography might be a method for separation and identification, but a difficulty was the lack of any means of detection of the spots. Several colour reactions found in the literature were tried, but were not found reliable. It was clear that a new method had to be worked out, and the following procedure was found to give good results with most derivatives tested.

After development, the dried paper is sprayed with $N / 2$ sodium hydroxide, and dried again. It is then brought in firm contact with a fluorescent screen and placed in front of an ultra-violet lamp, the paper facing the light. A low-pressure mercury lamp with a suitable filter letting through a fairly monochromatic light of about $2537 \mathrm{~A}$. and a screen which fluoresces in this light have been used. 'The acids are seen as dark spots on the fluorescent screen. The method proved to be very sensitive.

As the absorption of the barbiturates is highest at a $p H$ between 9 and 10, the spraying of the paper with an alkaline solution is very important. The resultant alkalinity of the paper is not known, and experiments are in progress to investigate which alkaline solution is to be preferred.

This arrangement of a screen which fluoresces with ultra-violet light seems to be a sensitive method for the detection of all kinds of substances absorbing ultra-violet light, and probably it has wide applications. Permanent records can, of course, be made in the usual way, by taking photographic contact prints of the sprayed and dried paper. It is, however, more convenient to draw pencil marks around the spots on the paper. After copying the drawing, the spots can be extracted if desired. By using this method it was found that the different barbituric acids could be separated by paper chromatography.

We are continuing to investigate this method and a full account will appear in the near future.

$$
\begin{gathered}
\text { Central Clinical Laboratory, } \\
\text { St. Erik's Hospital, } \\
\text { Stockholm. } \\
\text { June I2. }
\end{gathered}
$$

Arne Grieg

\section{Specificity of Vitamin $B_{12}$ for Euglena}

$I_{N}$ an earlier publication ${ }^{1}$ we reported that vitamin $\mathrm{B}_{12}$ could not be replaced for Euglena gracilis var. bacillaris by vitamin-free casein hydrolysate, carbontreated peptone, methionine, ascorbic acid, pteroylglutamic acid, a variety of purine and pyrimidine bases or by xanthopterin and a number of related compounds. Hutner et al. ${ }^{2}$ found thymidine to be ineffective. Vitamin $\mathrm{B}_{12 a}$ (hydroxocobalamin) was active $^{1}$. We have examined a number of additional compounds for vitamin $\mathrm{B}_{12}$ activity on Euglena.

Emerson et al. ${ }^{3}$ reported the vitamin $B_{12}$-like activity of $\alpha$ - and $\beta$-ribazole and some related compounds. Samples of these compounds were obtained through the courtesy of Dr. Karl Folkers and tested on Euglena in our liquid medium I 1,4. At 0.166 mgm. per ml., $\alpha$ - and $\beta$-ribazole had an activity on Euglena equivalent to $1-3 \cdot 4 \mu \mu \mathrm{gm}$. of vitamin $\mathrm{B}_{12}$ per $\mathrm{ml}$. At $0.00166 \mathrm{mgm}$. and $0.0000166 \mathrm{mgm}$. per $\mathrm{ml}$. no effect was observed. For Euglena, vitamin $\mathrm{B}_{12}$ was from $50,000,000$ to $150,000,000$ times as active as 